\title{
Efeitos de restrições ambientais na habilidade rebater em crianças, adultos e idosos
}

\author{
Ernani Filho \\ Roberto Gimenez \\ Cássio Júnior
}

https://doi.org/10.5628/rpcd.03.03.43

\author{
Escola de Educação Física e Esporte \\ Universidade de São Paulo, Brasil
}

\section{RESUMO}

Assumindo que o comportamento motor é um processo sujeito a mudanças e adaptações, o objetivo desse estudo foi investigar o efeito de diferentes condições sobre o padrão fundamental rebater em diferentes faixas etárias. Participaram da pesquisa cinco crianças, cinco adultos e cinco idosos, todos do sexo masculino. A tarefa foi a rebatida de força numa bola de plástico a uma parede, por meio de um bastão de madeira, em duas situações: (i) bola estática e (ii) bola em movimento. Foram feitos registros cinematográficos na posição perpendicular de cinco tentativas de cada sujeito em cada situação. Para tratamento dos dados recorreu-se aos testes de Kruskall Wallis e Wilcoxon com base nos valores da lista de checagem para análise do rebater adaptada de Williams (26). Não houve diferenças significativas entre as faixas etárias, quer seja na situação estática ou na situação em movimento. Entretanto, ocorreram algumas diferenças descritivas importantes: por exemplo, as crianças apresentaram uma tendência de modificação da ação para níveis inferiores de todos os componentes, da primeira situação para a segunda, porém com manutenção dos níveis de acerto.

\author{
ABSTRACT \\ Environmental constraints on striking: \\ children, adults and elderly people
}

Considering motor behavior as an adaptive and changing process, the aim of this work was to investigate the effect of environmental constraints in the fundamental motor pattern striking in different age groups. Five children, five adults and five elderly, all males, took part in the experiment. The experimental task was to strike a plastic ball as hard as possible against a wall, by means of a wood stick in two conditions: (i) static ball and (ii) moving ball. Five trials of each participant on each condition were registered with a video camera, in perpendicular position. Kruskall Wallis and Wilcoxon procedures were used to analyse data based on a checklist adapted from Williams (26). There were no differences among age groups on both conditions. However, there were important descriptive differences: for instance, children showed a tendency to change (to inferior levels) in the action of all components from the first to the second condition, but maintaining performance.

Key Words: striking, environmental constraints, motor behavior.

Palavras-chave: rebater, restrições ambientais, comportamento motor. 


\section{INTRODUÇÃO}

O desenvolvimento motor é visto como um processo seqüencial, contínuo e progressivo. Esse processo é identificado por um conjunto de etapas que se sucedem e que refletem níveis ou graus diferentes de habilidade motora (7). Cada etapa é identificada por um estágio particular de organização do comportamento motor (13).

Existem vários modelos que procuram descrever a seqüência de desenvolvimento motor $(7,13,20,21)$. Esses modelos sintetizam os principais achados de estudos descritivos longitudinais sobre o comportamento motor de bebês, crianças e adolescentes. Grande parte dos estudos realizados no início do século XX procurou caracterizar o comportamento motor de bebês e crianças de primeira infância (3). Trata-se de estudos longitudinais, cuja preocupação era descrever detalhadamente as etapas sucessivas que os bebês atravessavam em direção a um maior controle postural, na locomoção, e em tarefas de manipulação de objetos. A causa para as mudanças evidenciadas no comportamento das crianças era atribuída fundamentalmente a fatores relacionados ao processo maturacional, como sistema muscular e neurológico. Principalmente a partir da década de 1950, houve um envolvimento maior de profissionais de Educação Física com os estudos sobre o desenvolvimento motor. Mais especificamente, esse envolvimento resultou num volume maior de trabalhos, cujo enfoque concentrava-se sobre o comportamento de crianças de segunda infância e adolescente. Em particular, esses estudos procuravam levantar dados relacionados à maturação músculo-esquelética e correlacionálos com mudanças na performance motora $(6,8)$. Nos anos 70 do século passado, com o advento da abordagem de processamento de informação por parte da área de Psicologia, uma das preocupações presentes nos estudos em desenvolvimento motor passou a ser não somente a descrição das mudanças que aconteciam no comportamento, mas estabeleceu-se uma discussão aprofundada dos aspectos que dariam suporte a essas mudanças. Em outras palavras, a área de desenvolvimento motor deixou de se preocupar eminentemente com "o que muda" para procurar investigar com maior profundidade "o porquê das mudanças" (4). Outro aspecto a ser destacado é que a importância hegemônica da corrente maturacionista passa a dar lugar à corrente interacionista (maturação e experiência) no processo de desenvolvimento motor.

A década de 1980 foi marcada pelo surgimento da abordagem dos sistemas dinâmicos, a qual, norteada por preceitos da física, contribuiu de maneira significa para estudos relacionados ao controle e desenvolvimento motor (22). A explicação para as mudanças, até então restrita à maturação ou à capacidade de processar informação, passou a ser atribuída também a outros elementos, tais como restrições no ambiente e na tarefa, além de outras inerentes ao próprio organismo.

Nas últimas duas décadas do século XX, vários estudos procurando manipular as restrições do ambiente e da tarefa têm sido realizados. É mais especificamente sobre a relação entre essas restrições e os padrões motores que se pretende tratar na próxima seção. Lançando mão do conceito de restrições, Newell (16) preconiza que, além da maturação das estruturas internas do sistema nervoso central, outros componentes seriam também responsáveis pela emergência e organização de novos padrões de comportamento motor. As restrições seriam então as responsáveis pelo delineamento de possíveis configurações de um sistema. Ainda segundo Newell (16), as restrições seriam originárias: a) do organismo, b) do ambiente e c) da tarefa. As restrições no organismo podem ser estruturais (por exemplo, peso corporal, estatura, proporções de segmentos corporais, etc.) e/ou funcionais (por exemplo, maturação do sistema nervoso, motivação, etc.). As restrições ambientais são aquelas presentes no local onde a ação está sendo executada, como as forças físicas externas ao sujeito (força de gravidade, iluminação, calor, densidade do meio onde o indivíduo se encontra, etc.). As restrições da tarefa dizem respeito a imposições sobre a forma da execução, para que seja possível alcançar o objetivo da tarefa. Newell (16) classificou as restrições na tarefa em três categorias: a) meta da tarefa; b) regras que impõem especificações ou restringem a dinâmica das respostas e c) implementos ou máquinas que restringem a dinâmica das respostas. Atuando nos graus de liberdade desse sistema, essas restrições desencadeariam um processo de alterações no controle, o que favoreceria, de certa forma, a emergência de novos padrões de movimento diferenciados. 
O impacto das restrições na tarefa em interação com as restrições do organismo no processo de desenvolvimento tem sido relativamente pouco estudado. Entretanto, alguns estudos já começaram a mapear as influências das restrições ambientais na organização das habilidades motoras básicas $(9,15,17,19)$. Ainda que seja possível reconhecer a importância da coordenação e do controle nas ações habilidosas, nem todas as correntes que explicam o desenvolvimento motor atribuem o mesmo valor às restrições na construção dos padrões de coordenação.

Oliveira (17) afirma que, com as restrições, o papel da prescrição exercido pelos programas motores é minimizado, uma vez que elas atuariam na redução dos graus de liberdade do sistema para torná-lo mais eficiente e manejável no âmbito do controle. As alterações nessas restrições conseguem gerar, de uma forma indireta, mudanças nos padrões de coordenação que, por sua vez, acarretarão mudanças desenvolvimentistas.

Contudo, quando se trata de associar restrições ao desenvolvimento, é importante que se faça uma distinção na maneira como as restrições atuam entre duas situações: (a) restrições que criam condições ou dão suporte para o sistema apresentar um padrão de coordenação de uma capacidade latente ou já existente que, no entanto, desmantela-se, uma vez retirada ou modificada a restrição, e (b) restrições que geram novos padrões, os quais permanecem mesmo quando elas deixam de exercer influência. A primeira forma de atuação pode ser ilustrada pelo estudo de Von Hofsten (24). Nesse trabalho, bebês com idade variando entre seis e oito meses foram colocados em uma cadeira especial que fixava o tronco da criança, permitindo que a postura fosse controlada artificialmente. Essas condições aumentaram a frequiência de movimentos manuais para a interceptação de uma bola em movimento. Entretanto, quando os bebês foram retirados desse aparato e retornaram à posição de decúbito dorsal, a freqüência e a qualidade de movimentos manuais diminuíam, ou seja, o experimentador conseguiu "criar" um nível de desenvolvimento avançado no sub-sistema postura, o que levou à emergência de comportamentos organizados de busca e preensão manual de objetos. Com relação à segunda situação, pode-se citar estudos sobre a locomoção em bebês. A partir dos anos 80 do século XX, passou a ser reconhecida a capacidade de bebês que não se locomovem em apresentarem comportamento de passadas alternadas quando colocados e sustentados pela cintura numa esteira motorizada. Num experimento realizado com nove bebês, a partir de um mês de idade, o estímulo para a marcha foi dado com alterações na velocidade da esteira (23). Como resultado, todas as crianças testadas apresentaram rápida melhora na performance entre o terceiro e o sexto mês, demonstrando também melhora nos ajustamentos na coordenação das pernas quando submetidas a diferentes velocidades. Esse experimento mostrou que a estimulação da marcha automática em esteiras favoreceria o aparecimento da marcha independente entre o sexto e o décimo segundo meses. O desenvolvimento poderia ser caracterizado pelo aumento da variabilidade e sensibilidade quando da introdução das restrições e sua posterior retirada (23).

Oliveira e Manoel (18) investigam o comportamento do arremesso, em que crianças foram colocadas em duas situações: arremessar a distância e a um alvo. Os resultados indicaram que a mudança de objetivo da tarefa, - de arremesso a distância para arremesso ao alvo - ocasionou uma modificação descendente nos níveis de desenvolvimento dos componentes do arremesso na maioria dos sujeitos, embora isso não tenha ocorrido de forma mais marcante nas crianças mais avançadas (padrão maduro). Isso explicaria, por exemplo, o baixo desempenho (escore obtido) dessas crianças no arremesso ao alvo. Elas acabaram utilizando um padrão de arremesso pouco ajustado, reduzindo poucos graus de liberdade frente às demandas de precisão na tarefa ao alvo. Esse resultado indica alguns pontos que merecem atenção. Primeiro, as crianças com um padrão avançado no arremesso a distância, apresentaram um padrão inadequado para arremesso ao alvo. Isso sugere que o estágio maduro no qual eles foram classificados está superestimado. Segundo, a alteração na restrição da tarefa (de distância para alvo) não foi suficiente para modificar o padrão de arremessar na dimensão exigida. Esses aspectos indicam que a construção da restrição interna, expressa na formação de um programa de ação, pode desempenhar um papel crucial no 
desenvolvimento motor. A crescente influência da abordagem dos sistemas dinâmicos fez com que o papel de representações mentais fosse minimizado ao extremo. Talvez esse total abandono seja, no mínimo, prematuro.

Barela e Barela (1) propõem que uma maneira de examinar como as restrições estão de fato influenciando o comportamento motor seja verificar o grau de estabilidade dos padrões observados. $\mathrm{O}$ aumento ou a diminuição da estabilidade do comportamento estaria associado a momentos diferentes no desenvolvimento: maior instabilidade no comportamento é indicativo de que o indivíduo está prestes a transitar para um novo estado de desenvolvimento. Comentando sobre a capacidade dos sujeitos para modificar o comportamento em face das restrições, Manoel e Pellegrini (14) apontam que essa capacidade estaria relacionada ao estágio de desenvolvimento motor dos sujeitos. Aqueles que estivessem em níveis mais avançados teriam maiores condições de manipular os seus graus de liberdade e, por conseguinte, de promover mais ajustes no comportamento. Visão semelhante do problema é oferecida por Langendorfer $(9,10)$ e Roberton (19) quando comentam as dificuldades que os iniciantes apresentam para ajustar os seus comportamentos.

Em linhas gerais, os dados presentes na literatura ainda não permitem retratar claramente o grau de influência das restrições nos padrões motores. Esse problema torna-se ainda maior quando se leva em consideração que os estudos sobre o processo de desenvolvimento motor, em sua maioria, restringem-se ao comportamento de crianças e adolescentes. São escassos os estudos envolvendo amostras de adultos e idosos.

O presente trabalho teve o intuito de estudar de forma exploratória o padrão fundamental rebater de indivíduos de diferentes faixas etárias, com base nas restrições introduzidas no ambiente.

A habilidade motora do rebater é identificada por um impulso proporcionado a um objeto, dependa ele ou não do uso de um implemento, e acontece num curto período de tempo para o contato. Williams (26) destaca que a habilidade motora chutar pode ser enqua- drada no grande grupo de habilidades de rebater, pois reúne características desse tipo de habilidade. Contudo, de acordo com a autora, essa habilidade, pela própria natureza do padrão de movimento, merece um tratamento em separado. Afirmação semelhante pode ser feita em relação ao saque do voleibol.

Tradicionalmente, a habilidade rebater tem sido descrita a partir do uso de um implemento $(7,25,26)$. Sugere-se que a execução dessa habilidade motora seja muito dependente de capacidades motoras, como tempo de reação, equilíbrio, tempo de movimento, e timing coincidente. Nesse sentido, com o decorrer do processo de desenvolvimento na infância, uma possível melhora dessas capacidades pode estar associada, em maior ou menor grau, a mudanças na qualidade da execução dessa habilidade. Williams (26) aponta que, com o processo de desenvolvimento, é esperado que o indivíduo realize a tarefa com um menor envolvimento dos graus de liberdade. Outro aspecto crucial diz respeito a uma tendência por parte do indivíduo que se encontra em estágios iniciais a assumir um padrão vertical para a ação, sobretudo no tocante ao movimento do tronco, braço e antebraço. Por outro lado, indivíduos que se encontram em estágios mais avançados tendem a apresentar uma rotação mais pronunciada do tronco, aliada a um movimento horizontalizado do braço e antebraço no lado onde se encontra o implemento. O mesmo autor apresenta uma lista de checagem para a análise da tarefa rebater (Quadro 1). Essa lista leva em consideração a ação dos componentes tronco e posição da cabeça, braço, perna e o controle geral do corpo, sendo que cada um dos componentes do corpo apresenta uma série de níveis que denotam a qualidade da execução.

A lista a seguir, apresenta os componentes divididos em níveis, identificados por letras (a, b, c, d, etc). É fundamental destacar que níveis mais avançados são identificados respectivamente por letras posteriores do alfabeto. Assim, por exemplo, (b) é mais avançado que (a) e (c) é mais avançado que (a) e (b). Um indivíduo pode ser considerado maduro quando apresenta todos ou a maioria dos componentes em níveis avançados. 
Quadro 1 - Lista de checagem para a análise do rebater com implemento (adaptada de Williams, 1983).

\section{AÇÃO DO TRONCO E POSIÇÃO DA CABEÇA}

a) o foco e o rosto se concentram no objeto a ser rebatido

b) o rosto é direcionado para o objeto e/ou direção do vôo pretendido

a) o corpo posiciona-se paralelamente ou na mesma linha do objeto a ser rebatido

b) o corpo se posiciona perpendicularmente à linha do vôo e é feita uma rotação em direção ao vôo pretendido no "swing" para traz

a) corpo posicionado muito próximo ao objeto

b) corpo posicionado a uma distância apropriada do objeto

\section{AÇÃO DO BRAÇO}

\section{Fase de preparação}

a) o atraso do braço ocorre na altura da cabeça

b) o atraso do braço é horizontal e o cotovelo mantido

longe do corpo
a) implemento apoiado no ombro
b) implemento apoiado fora do ombro

\section{AÇÃO DO BRAÇO}

\section{Fase de ação}

a) o movimento se deve fundamentalmente a uma ação do ombro

b) o movimento se deve fundamentalmente a uma ação lateral do braço num plano horizontal e o cotovelo é estendido durante o balanço para frente e o contato com o objeto é feito com o braço estendido

\section{AÇÃO DA PERNA}

a) pés posicionados próximos um do outro

b) pés na largura do ombro
a) não há transferência de peso
b) o peso é transferido para a perna de trás durante o balanço
c) o peso é transferido para a perna de trás durante o balanço e um passo é dado em direção à linha de vôo pretendida

\section{CONTROLE DO CORPO}

a) o corpo move-se como uma unidade

b) o movimento é suave e seqüencial com a ação sendo iniciada pelo tronco
Convencionalmente, sugere-se que crianças norteamericanas de seis anos de idade já apresentariam estágios avançados para rebater uma bola estática com implemento. Todavia, é possível que essas crianças apresentem níveis inferiores diante de mudanças introduzidas no ambiente, como, por exemplo, nos casos em que a bola estiver em movimento. De maneira semelhante, dúvidas persistem em relação ao comportamento de adultos e idosos. Especialmente em relação a esses últimos, é possível que restrições organísmicas impliquem dificuldades para executar um padrão em particular. O propósito do presente estudo é investigar essas possíveis diferenças no comportamento dos três grupos - crianças, adultos e idosos - na tarefa motora rebater, realizada em diferentes condições.

As questões de estudo são as seguintes: 1) As mudanças nas condições do ambiente resultam em mudanças nos padrões de rebater dos grupos? 2) Existem diferenças nos padrões de rebater empregados pelos grupos nas diferentes situações?

\section{MATERIAL E MÉTODOS \\ Amostra}

Participaram desse estudo 15 sujeitos divididos em três grupos: grupo 1, constituído por cinco crianças (média de idade 5,5 anos); grupo 2, constituído por cinco adultos (média de idade 24,4 anos); e grupo 3, constituído por cinco indivíduos idosos (média de idade 69,2 anos). Todos os sujeitos eram do sexo masculino destros, os adultos e os idosos participavam de programas formais de atividade física da Escola de Educação Física e Esporte da Universidade de São Paulo, já as crianças participavam das aulas regulares de educação física na pré-escola.

\section{Metodologia}

A tarefa utilizada foi a rebatida de uma bola de plástico (10 cm de diâmetro e peso de 155 g) a uma parede com a maior força possível, por meio de um bastão de madeira. Para idosos e adultos o bastão tinha um metro de comprimento; para as crianças, a medida do bastão correspondeu a 50 centímetros. A tarefa de rebater foi realizada em duas condições: (1) bola estática, posicionada sobre um cone plástico cuja altura correspondia aproximadamente à altura da cintura pélvica do sujeito, um metro para os adul- 
tos e idosos e 0,5 metros no caso das crianças; (2) bola em movimento, lançada por um experimentador, na linha média do corpo, na altura da cintura pélvica do indivíduo. Todos os indivíduos foram solicitados a rebater a bola o mais longe possível em cinco tentativas.

Foram feitos registros cinematográficos na posição frontal por meio de uma câmera Super VHS (Panasonic), posicionada em frente a cada sujeito à distância de aproximadamente cinco metros. A distância do experimentador para o sujeito também correspondeu a cinco metros.

\section{Análise dos dados e tratamento estatístico}

Para a análise dos dados, tomou-se como base uma lista de checagem do rebater adaptada de Williams (26) (apresentada anteriormente). Com o intuito de quantificar objetivamente a consistência do padrão apresentado pelos grupos, estabeleceu-se um índice de consistência (IC) para o padrão dos sujeitos. Esse índice foi calculado a partir da seguinte equação:

\section{IC = Moda [número de tentativas em que se repetiu o padrão]}

Número total de tentativas

Procurou-se também estimar o grau de acerto dos indivíduos ao longo das tentativas. Para tanto, estabeleceu-se um índice de acerto (IA), que representa, no número total de tentativas, o percentual daquelas em que os indivíduos conseguiram acertar a bola. Para verificar o padrão de execução, nas duas situações, empregou-se a moda para a ação de cada componente isoladamente. Para verificar a existência de possíveis diferenças no Índice de Consistência entre os grupos e entre as tarefas, utilizou-se o teste não paramétrico de Wilcoxon. Para verificar a existência de possíveis diferenças nos Índices de Acerto, empregou-se uma análise de variância não paramétrica Kruskall Wallis.

\section{RESULTADOS}

Os grupos não apresentaram diferenças na ação dos componentes na situação 1. Em outras palavras, na tarefa em que a bola se encontrava estática, os grupos apresentaram padrões de rebater similares. Tendo como base a moda, o padrão dos grupos caracterizou-se por: um posicionamento da cabeça voltado para a direção do vôo pretendido para a bola; corpo posicionado perpendicularmente à linha do vôo, com a realização de um swing para trás em preparação; corpo posicionado a uma distância apropriada da bola; atraso do braço na horizontal com implemento apoiado fora do ombros; pés posicionados na largura da linha do ombro; com transferência de peso da perna de trás durante o balanço para a perna da frente, e movimento seqüencial e iniciado pela ação do tronco. As diferenças aconteceram em relação à ação do braço, pois uma criança e dois adultos não estenderam o cotovelo durante a execução e na transferência de peso para a perna de trás, na fase de balanço, que não foi realizada por dois sujeitos do grupo de adultos e por dois sujeitos do grupo de idosos. Vale ainda ressaltar que o movimento seqüencial iniciado pelo tronco aconteceu em apenas três crianças, três adultos e quatro idosos.

Os dados encontrados junto aos grupos na condição 1 foram agrupados na Tabela 1. 
Tabela 1 - Ação dos componentes na tarefa de rebater a bola estática em crianças, adultos e idosos (a letra "S" da tabela significa "sujeito"]

\begin{tabular}{|c|c|c|c|}
\hline Ação do Tronco e posição da cabeça & Crianças & Adultos & Idosos \\
\hline Foco e rosto no objeto & - & - & - \\
\hline Rosto direcionado ao vôo pretendido & $\mathrm{S} 1, \mathrm{~S} 2, \mathrm{~S} 3, \mathrm{~S} 4$ e S5 & $\mathrm{S} 1, \mathrm{~S} 2, \mathrm{~S} 3, \mathrm{~S} 4$ e S5 & $\mathrm{S} 1, \mathrm{~S} 2, \mathrm{~S} 3, \mathrm{~S} 4$ e S5 \\
\hline Corpo posicionado paralelamente ou na mesma linha do objeto & - & - & - \\
\hline $\begin{array}{l}\text { Corpo posicionado perpendicularmente à linha do vôo, } \\
\text { com rotação em direção ao vôo pretendido }\end{array}$ & S1,S2, S3, S4 e S5 & S1,S2, S3, S4 e S5 & $\mathrm{S} 1, \mathrm{~S} 2, \mathrm{~S} 3, \mathrm{~S} 4$ e S5 \\
\hline Corpo posicionado próximo ao objeto & - & - & - \\
\hline Corpo a uma distância apropriada do objeto & $\mathrm{S} 1, \mathrm{~S} 2, \mathrm{~S} 3, \mathrm{~S} 4$ e S5 & S1,S2, S3, S4 e S5 & $\mathrm{S} 1, \mathrm{~S} 2, \mathrm{~S} 3, \mathrm{~S} 4$ e S5 \\
\hline Ação do braço [fase de preparação] & Crianças & Adultos & Idosos \\
\hline Atraso na altura da cabeça & - & - & - \\
\hline Atraso na horizontal e cotovelo longe do corpo & $\mathrm{S} 1, \mathrm{~S} 2, \mathrm{~S} 3, \mathrm{~S} 4$ e S5 & $\mathrm{S} 1, \mathrm{~S} 2, \mathrm{~S} 3, \mathrm{~S} 4$ e S5 & $\mathrm{S} 1, \mathrm{~S} 2, \mathrm{~S} 3, \mathrm{~S} 4$ e S5 \\
\hline Ação do braço [fase de ação] & Crianças & Adultos & Idosos \\
\hline 0 movimento se deve a uma ação do ombro & S2 & S3 e S4 & - \\
\hline $\begin{array}{l}0 \text { movimento se deve a uma ação do braço na horizontal e o } \\
\text { cotovelo é estendido no balanço }\end{array}$ & $\mathrm{S} 1, \mathrm{~S} 3, \mathrm{~S} 4$ e S5 & S1,S2, e S5 & $\mathrm{S} 1, \mathrm{~S} 2, \mathrm{~S} 3, \mathrm{~S} 4$ e S5 \\
\hline Ação da perna & Crianças & Adultos & Idosos \\
\hline Pés próximos um do outro & - & - & - \\
\hline Pés na largura do ombro & $\mathrm{S} 1, \mathrm{~S} 2, \mathrm{~S} 3, \mathrm{~S} 4$ e S5 & $\mathrm{S} 1, \mathrm{~S} 2, \mathrm{~S} 3, \mathrm{~S} 4$ e S5 & $\mathrm{S} 1, \mathrm{~S} 2, \mathrm{S3}, \mathrm{S} 4$ e S5 \\
\hline Não há transferência de peso & - & S1 e S5 & S4 e S5 \\
\hline 0 peso é transferido para a perna de trás & $\mathrm{S} 1, \mathrm{~S} 2, \mathrm{~S} 3, \mathrm{~S} 4$ e S5 & $\mathrm{S} 2, \mathrm{~S} 3$ e S4 & $\mathrm{S} 1, \mathrm{~S} 2, \mathrm{~S} 3$ \\
\hline $\begin{array}{l}\text { O peso é transferido para a perna de trás e um passo é dado } \\
\text { em direção à linha de vôo }\end{array}$ & - & - & - \\
\hline Controle do corpo & Crianças & Adultos & Idosos \\
\hline Corpo move-se como uma unidade & S2 e S5 & S2 e S4 & S4 \\
\hline 0 movimento é suave e seqüencial e a ação é iniciada pelo tronco & S1, S3 e S4 & S1, S3, e S5 & $\mathrm{S} 1, \mathrm{~S} 2, \mathrm{~S} 3$ e S5 \\
\hline
\end{tabular}

Na condição 2, a moda indica que o padrão característico dos três grupos também é identificado por: um posicionamento da cabeça voltado para a direção do vôo pretendido para a bola; corpo posicionado perpendicularmente à linha do vôo, com a realização de swing para trás em preparação; posicionamento do corpo a uma distância apropriada da bola; atraso do braço na horizontal e pés posicionados na largura da linha do ombro. Um diferencial em relação à condição 1, é que, em sua maioria, os indivíduos dos três grupos apresentaram redução na transferência de peso da perna de trás para a da frente e na rotação iniciada pelo tronco.

Os dados encontrados junto aos grupos na condição 2 foram agrupados na Tabela 2. 
Tabela 2 - Ação dos componentes na tarefa de rebater a bola lançada em crianças, adultos e idosos.

\begin{tabular}{|c|c|c|c|}
\hline Ação do Tronco e posição da cabeça & Crianças & Adultos & Idosos \\
\hline Foco e rosto no objeto & - & - & - \\
\hline Rosto direcionado ao vôo pretendido & $\mathrm{S} 1, \mathrm{~S} 2, \mathrm{~S} 3, \mathrm{~S} 4$ e S5 & $\mathrm{S} 1, \mathrm{~S} 2, \mathrm{~S} 3, \mathrm{~S} 4$ e $\mathrm{S} 5$ & $\begin{array}{l}\mathrm{S} 1, \mathrm{~S} 2, \mathrm{~S} 3, \mathrm{~S} 4 \mathrm{e} \\
\mathrm{S} 5\end{array}$ \\
\hline Corpo posicionado paralelamente ou na mesma linha do objeto & S1 e S5 & - & - \\
\hline $\begin{array}{l}\text { Corpo posicionado perpendicularmente à linha do vôo, } \\
\text { com rotação em direção ao vôo pretendido } \\
\text { Corpo posicionado próximo ao objeto }\end{array}$ & $\begin{array}{c}\mathrm{S} 2, \mathrm{~S} 3 \text { e S4 } \\
- \\
-\end{array}$ & $\begin{array}{c}\mathrm{S} 1, \mathrm{~S} 2, \mathrm{~S} 3, \mathrm{~S} 4 \text { e S5 } \\
-\end{array}$ & $\begin{array}{c}\text { S1,S2, S3, S4 e } \\
\text { S5 } \\
-\end{array}$ \\
\hline Corpo a uma distância apropriada do objeto & S1,S2, S3, S4 e S5 & S1,S2, S3, S4 e S5 & $\begin{array}{l}\text { S1,S2, S3, S4 e } \\
\text { S5 }\end{array}$ \\
\hline Ação do braço (fase de preparação] & Crianças & Adultos & Idosos \\
\hline Atraso na altura da cabeça & $\mathrm{S} 1, \mathrm{~S} 3$ e S5 & - & - \\
\hline Atraso na horizontal e cotovelo longe do corpo & $\mathrm{S} 2$ e S4 & $\mathrm{S} 1, \mathrm{~S} 2, \mathrm{~S} 3, \mathrm{~S} 4$ e S5 & $\begin{array}{c}\mathrm{S} 1, \mathrm{~S} 2, \mathrm{~S} 3, \mathrm{~S} 4 \mathrm{e} \\
\mathrm{S} 5\end{array}$ \\
\hline Ação do braço [fase de ação] & Crianças & Adultos & Idosos \\
\hline 0 movimento se deve a uma ação do ombro & S1, S2, S3 e S5 & S4 & S5 \\
\hline $\begin{array}{l}0 \text { movimento se deve a uma ação do braço na horizontal } \\
\text { e o cotovelo é estendido no balanço }\end{array}$ & S4 & $\mathrm{S} 1, \mathrm{~S} 2, \mathrm{~S} 3$ e $\mathrm{S} 5$ & $\mathrm{~S} 1, \mathrm{~S} 2, \mathrm{~S} 3, \mathrm{e}$ S4 \\
\hline Ação da perna & Crianças & Adultos & Idosos \\
\hline Pés próximos um do outro & S3, S4 e S5 & - & - \\
\hline Pés na largura do ombro & S1 e S2 & S1,S2, S3, S4 e S5 & $\begin{array}{l}\mathrm{S} 1, \mathrm{~S} 2, \mathrm{~S} 3, \mathrm{~S} 4 \mathrm{e} \\
\mathrm{S} 5\end{array}$ \\
\hline Não há transferência de peso & $\mathrm{S} 1, \mathrm{~S} 2, \mathrm{~S} 3, \mathrm{~S} 4 \mathrm{e}$ S5 & S1 e S5 & S3, S4 e S5 \\
\hline 0 peso é transferido para a perna de trás & - & $\mathrm{S2}, \mathrm{S3}$ e $\mathrm{S} 4$ & S1 e S2 \\
\hline $\begin{array}{l}0 \text { peso é transferido para a perna de trás e um passo é dado em } \\
\text { direção à linha de vôo }\end{array}$ & - & - & - \\
\hline Controle do corpo & Crianças & Adultos & Idosos \\
\hline Corpo move-se como uma unidade & S1, S2 e S4 & S2, S4 e S5 & S3,S4 e S5 \\
\hline 0 movimento é suave e seqüencial e a ação é iniciada pelo tronco & S3 e S5 & S1 e S3 & $\mathrm{S} 1 \mathrm{e}$ S2 \\
\hline
\end{tabular}

No tocante aos índices de consistência, procurou-se agrupar os dados com base na ação de cada componente (tronco/posição da cabeça; ação do braço; ação da perna e controle do corpo), uma vez que eles variaram em taxas diferentes entre os grupos. A Figura 1 apresenta os dados correspondentes ao IC no grupo das crianças nas Condições 1 e 2 . 


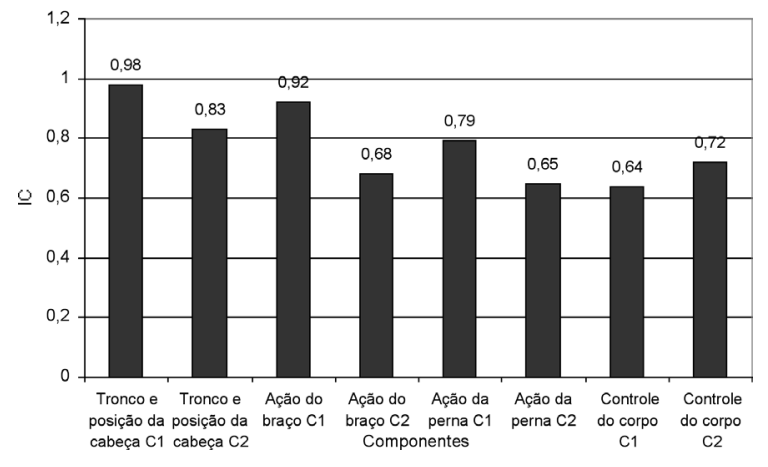

Figura 1 - Índice de consistência por componente na condição de bola parada e em movimento (grupo das crianças].

No grupo das crianças, a realização do teste nãoparamétrico de Wilcoxon não apontou diferenças significativas em qualquer dos componentes da condição 1 para a 2, ou seja, os correspondentes a esse grupo não permitem inferir que as restrições na tarefa foram suficientes para alterações no padrão de execução. Todavia, conforme pode ser observado na Figura 1, foi o componente ação do braço que diminuiu mais o IC da condição 1 para a 2 .

A Figura 2 reúne os dados correspondentes ao grupo dos adultos.

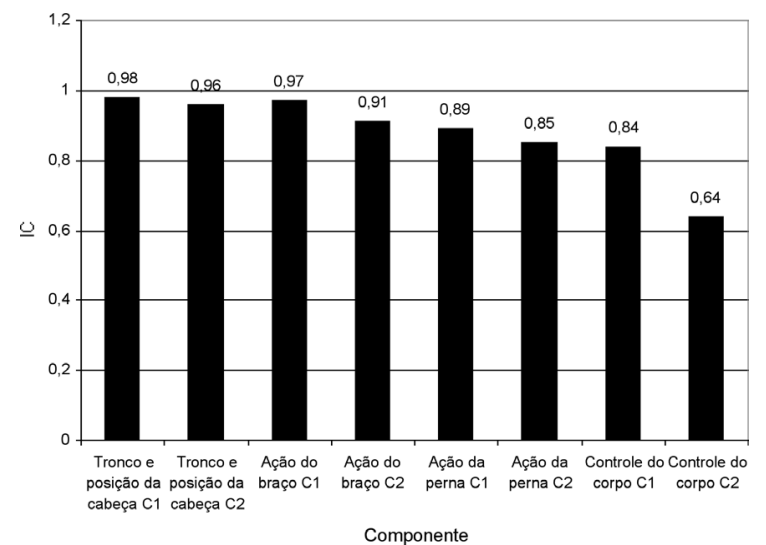

Figura 2 - Índice de consistência por componente na condição de bola parada e em movimento (grupo dos adultos).
De acordo com a Figura 2, pode perceber-se que houve uma tendência geral de diminuição do IC, no grupo de adultos, com as mudanças introduzidas nas condições da tarefa. No entanto, não foram encontradas diferenças estatisticamente significativas de acordo com o teste não-paramétrico de Wilcoxon. Finalmente, os dados correspondentes ao IC do grupo de idosos estão representados na Figura 3.

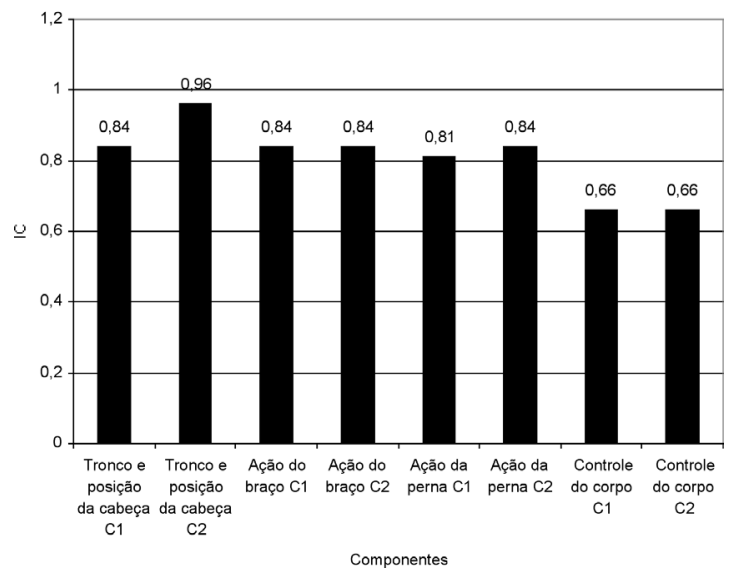

Figura 3 - Índice de consistência por componente na condição de bola parada e em movimento (grupo dos idosos].

Da mesma forma como aconteceu com os demais grupos, não foram encontradas diferenças estatisticamente significativas da condição 1 para a 2 , com base no teste $\mathrm{d}$ Wilcoxon. Ainda, no tocante ao índice de acerto (IA), a realização da análise de variância para dados não paramétricos Kruskal Wallis também não apontou diferenças entre os grupos. Os dados correspondentes ao IA nas duas condições estão representados na Figura 4. 


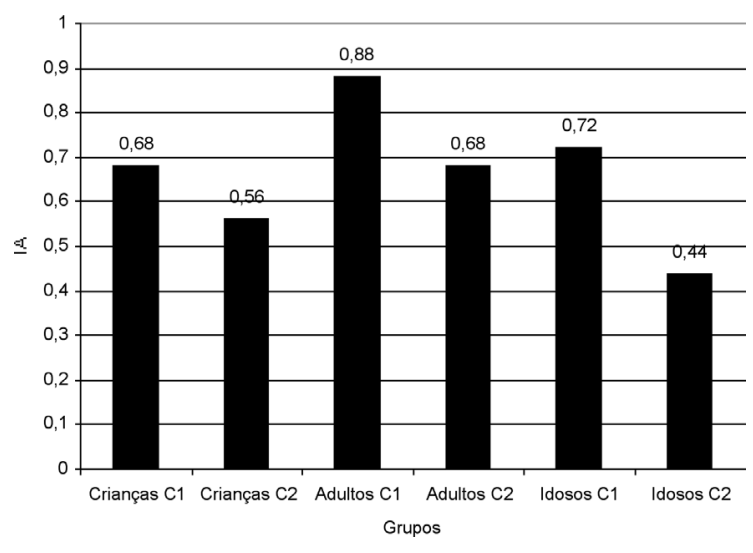

Figura 4 - Índice de acerto das crianças, adultos e idosos na condição de bola parada e em movimento.

\section{DISCUSSÃO}

Não foram encontradas diferenças entre os três grupos, sobretudo na condição de bola estática.

Evidenciaram-se apenas alguns comportamentos diferenciados por parte de certos indivíduos. Isso aconteceu sempre num componente em particular, como por exemplo: na ação do braço (uma criança e dois adultos); na transferência de peso (dois adultos e dois idosos); e no controle do corpo (duas crianças, dois adultos e um idoso).

Também não houve diferenças pronunciadas entre os grupos com a mudança nas condições do ambiente. Mais especificamente, isso pode ser percebido pela comparação dos índices de consistência das condições de bola estática e em movimento, que não sofreram mudanças significativas em nenhum dos grupos. Contudo, uma análise mais detalhada dos dados referentes à ação dos componentes indica que o grupo das crianças apresentou uma tendência geral em modificar a ação de alguns componentes com a mudança no ambiente. Essas alterações aconteceram em todos os componentes - ação do tronco e posição da cabeça, ação do braço, ação da perna, controle do corpo. No que diz respeito à ação do tronco e posição da cabeça, duas crianças alteraram o posicionamento perpendicular em relação ao vôo pretendido para a bola, adotando um posicionamento paralelo em relação a ela. No componente ação do braço, três crianças que realizavam um atraso na horizontal, manten- do o cotovelo longe do corpo na execução, passaram a atrasar o braço na altura da cabeça. Quatro crianças passaram também a concentrar a resposta ao objeto na ação mais concentrada no ombro, em vez de mantê-la numa ação integrada entre ombro e cotovelo. No tocante ao componente pernas, três crianças aproximaram os pés um do outro na ação. Todas as crianças deixaram de realizar a transferência de peso. Finalmente, em relação ao componente controle do corpo, três crianças passaram a movimentá-lo como uma unidade. É fundamental destacar que todas essas mudanças estão relacionadas a padrões mais inferiores para a execução. Em outras palavras, houve uma tendência por parte das crianças em apresentarem padrões mais rudimentares diante das mudanças nas condições do ambiente.

$\mathrm{Na}$ condição 1 (bola estática), todas as crianças apresentaram padrões avançados na ação da maioria dos componentes. Esses dados corroboram os pressupostos de Williams (26), que sugere que crianças enquadradas nessa faixa etária já apresentam um padrão avançado na habilidade rebater. No entanto, quando essas crianças foram expostas a uma condição que necessitava ajustes maiores em relação aos componentes do corpo e que se caracterizava por uma imprevisibilidade maior por parte do ambiente, houve uma tendência a recorrerem a níveis mais inferiores para a execução. Essas alterações podem ser consideradas como indicativos de que o sistema ainda não reúne as capacidades necessárias para uma execução adequada.

A execução de uma tarefa como a condição 2 apresenta uma demanda maior por parte de capacidades neuromotoras como, por exemplo, equilíbrio recuperado (no retorno do balanço) e timing coincidente (para acertar a bola) (12). É possível que crianças dessa faixa etária ainda não tenham essas capacidades bem desenvolvidas. Tendo em vista que essas capacidades dependem muito do sistema nervoso central, é provável que haja uma acentuada melhora das mesmas até o final da primeira década de vida $(5,6)$.

Um aspecto importante é que apesar das crianças serem afetadas pelas restrições da tarefa, elas demonstravam grande capacidade de adaptação. Em especial, isso pode ser percebido pelos dados referentes ao acerto da bola, que não apresentaram diferenças significativas da condição 1 para a 2 . 
Genericamente, os adultos mantiveram o padrão de execução da condição 1 para a 2. Algumas diferenças foram encontradas, particularmente na ação do componente controle do corpo, por parte de um sujeito, que passou a movê-lo como uma unidade. A tendência à estabilidade do comportamento também se apresenta nos índices de consistência desse grupo da condição 1 para a 2, uma vez que não houve diferenças estatisticamente significativas. Embora não tenham sido encontradas diferenças estatisticamente significativas nos índices de acerto, os dados encontrados sugerem que houve uma diminuição dos mesmos das condições 1 para a 2 . A maior imprevisibilidade da tarefa na condição 2 contribui para explicar a tendência de diminuição da eficácia no alcance do resultado da ação.

No tocante ao grupo de idosos, o padrão de execução tanto na condição 1 como na condição 2 , apresentou-se de maneira muito semelhante ao grupo de adultos. Em especial, nos componentes controle do corpo e ação das pernas, ocorreram alterações da condição 1 em relação à condição 2 . Dois indivíduos passaram a mover o tronco como uma unidade, e outro passou a não transferir peso da perna de trás para a da frente.

Esse conjunto de dados também sugere que as mudanças nas condições da tarefa não foram suficientes para promover alterações nos padrões. Os dados encontrados corroboram os de Williams et al. (27), que não identificaram diferenças estruturais na execução do padrão de arremesso em idosos, em comparação com adultos. É oportuno destacar ainda que, apesar de não terem sido encontradas diferenças estatisticamente significativas entre os índices de consistência da condição 1 para a condição 2 , em comparação aos demais grupos, os idosos foram os que apresentaram maior tendência em manter a consistência do padrão de movimento. Em outras palavras, esse foi o grupo que, ao adotar um padrão de resposta para a tarefa, mais o manteve ao longo das tentativas. É possível que, diante de algumas restrições organísmicas naturais do processo de desenvolvimento, o idoso venha a perder algumas capacidades neuromotoras, por exemplo, equilíbrio, agilidade, tempo de reação $(2,11)$.

Por outro lado, apesar da ausência de diferenças estatisticamente significativas, a análise dos dados correspondentes aos índices de acerto sugere que da condição 1 para a 2, em comparação às crianças e aos adultos, os idosos passaram a errar mais. Esse fator pode ser um indicador de que a busca pela manutenção do padrão pode ser prejudicial à eficácia da execução.

\section{CONSIDERAÇÕES FINAIS}

O presente estudo teve um caráter exploratório. O objetivo principal foi verificar os possíveis efeitos das restrições da tarefa em indivíduos de diferentes faixas etárias. Os resultados não indicam diferenças pronunciadas entre crianças, adultos e idosos na habilidade de rebater uma bola estática. Algumas diferenças no padrão de movimento dos três grupos apareceram quando a tarefa foi modificada para uma condição em que a bola era lançada pelo experimentador. Houve uma tendência por parte das crianças em recorrer a padrões mais rudimentares na condição de bola em movimento. Num outro extremo, o grupo de idosos foi o que pareceu alterar menos o padrão com as restrições impostas na tarefa. Tanto o padrão dos idosos como o das crianças pode ter sido influenciado pelas restrições organísmicas presentes nos dois grupos. Essas restrições organísmicas relacionam-se, sobretudo, à maturação do sistema nervoso central, que exerce muita influência sobre capacidades motoras como equilíbrio e timing coincidente. Esse estudo retoma a discussão sobre as possibilidades de adaptação e mudança do ser humano, em função dos desafios que se apresentam no meio ambiente. É importante que estudos futuros comparem o comportamento de grupos constituídos por diferentes faixas. Esses estudos podem fazer uso de instrumentos que possibilitem verificar diferenças quantitativas no padrão de movimento dos grupos, ou ainda, que usem uma lista de checagem mais detalhada. Vale ainda ressaltar a importância de serem realizados trabalhos sobre outras habilidades motoras e com amostras mais representativas. Estudos nessa direção podem contribuir para enriquecer a discussão sobre os fatores subjacentes às mudanças que acontecem no comportamento motor ao longo da vida. As seguintes conclusões são derivadas das considerações acima: 
- o padrão de movimento da rebatida de crianças, adultos e idosos não difere quando o objeto a ser interceptado encontra-se em posição estática;

- o padrão de movimento da rebatida de crianças, adultos e idosos difere quando o objeto a ser interceptado é lançado pelo experimentador; crianças recorrem a padrões mais rudimentares e idosos não arriscam variar o padrão;

- diferentes padrões de movimento nas diferentes faixas etárias podem estar relacionados à maturação do sistema nervoso central, que exerce muita influência sobre capacidades motoras como equilíbrio e timing coincidente.

\section{CORRESPONDÊNCIA}

\section{Ernani Xavier Filho}

Laboratório de Comportamento Motor (LACOM)

Escola de Educação Física e Esporte

Universidade de São Paulo

Avenida Professor Mello Moraes, 65

Cidade Universitária - São Paulo - SP

CEP: 05508-900 Brasil

piraju@usp.br

\section{REFERÊNCIAS BIBLIOGRÁFICAS}

1 Barela, A.M.; Barela, J.A. (1997). Restrições ambientais no arremesso de ombro. Motriz, 3: 65-72.

2 Binder, E.F.; Brown, M.; Craft, S.; Schecheman, K.; Birge, S.J. (1994). Effects of a group exercise program on risk factors for falls in frail older adults. Journal of Aging and Physical Activity, 2: 25-37.

3 Clark, J.E.; Whitall, J. (1989). What is motor development? The lessons of history. Quest, 41: 183-202.

4 Connolly, K.J. (1970). Skill development: problems and plans In: K.J. Connolly (Ed.). Mechanisms of motor skill development. London: Academic Press, 3-21.

5 Cratty, J.B. (1986). Acquiring Skill: Development Perspectives. In: J. B. Cratty (Ed.). Perceptual and motor development in infants and children. New Jersey, Englewood Cliffs, 146-57.

6 Eckert, H. (1993). Desenvolvimento motor. São Paulo: Manole.

7 Gallahue, D.; Ozmun, J.C. (2001). Compreendendo o desenvolvimento motor: bebês, crianças, adolescentes e adultos. São Paulo: Phorte Editora.

8 Guedes, D. P.; Guedes, J.E.R.P. (1997). Crescimento, composição corporal e desempenho motor em crianças e adolescentes. São Paulo: CLR Baliero.

9 Langendorfer, S.J. (1987). Prelongitudinal screening of overarm striking development performed under two environmental conditions. In: J. Clark, J.H. Humphrey (Eds.). Advances in motor development research-1. New York: MAS Press, 17-47.

10 Langendorfer, S.J. (1990). Contemporary Trends in infant preschool aquatics into the 1990s and beyond. JOPERD, 61: 36-39.

11 Macauley, E.; Mihalko, S.L.; Rosengren, K. (1997). Selfefficacy; and balance correlates of fear of failing in the elderly. Journal of Aging and Physical Activity, 5: 329-40.

12 Magill, R.A. (2000). Aprendizagem Motora: conceitos e aplicações. São Paulo: Edgard Blücher.

13 Manoel, E.J. (1994). Desenvolvimento motor: implicações para a educação física escolar. Revista Paulista de Educação Física, 8: 82-97.

14 Manoel, E.J.; Pelegrini, A.M. (1985). Evolução do padrão fundamental de movimento frente a duas tarefas: dados preliminares. In: Anais do Congresso Brasileiro de Ciências do Esporte. Minas Gerais, Poços de Caldas, 3-4.

15 Marques, I. (1995). Padrão fundamental de movimento: uma análise universal ou contextual? Dissertação. Faculdade de Educação Física, Universidade de Campinas. Campinas - SP.

16 Newell, K.M. (1986). Constraints on the development of coordination In: M. G. Wade, H.T.A. Whithing (Eds.). Motor development in children: aspects of coordination and control. Amsterdam: Martinus Nijhooff, 85-122.

17 Oliveira, J.A. (1997). Estado de desenvolvimento no padrão fundamental de movimento arremessar frente a variações numa restrição da tarefa. Dissertação. Escola de Educação Física e Esporte, Universidade de São Paulo. São Paulo - SP.

18 Oliveira, J.A.; Manoel, E.J. (2000). Motor developmental status and task constraint in overarm throwing. Journal of Human Movement Studies, 39: 358-78.

19 Roberton, A.M. (1987). Developmental level as a function of immediate environment. In: J. E. Clark, J. H. Humphrey 
(Eds.). Advances in motor development research-1. New York, AMS Press, 1-15.

20 Seaman, J.; DePauw, K. (1982). The new adapted physical education. California: Mayfield Publishing Company.

21 Tani, G.; Manoel, E.J.; Kobubun, E.; Proença, J.E. (1988). Educação física escolar: fundamentos para uma abordagem desenvolvimentista. São Paulo: EPU/EDUSP.

22 Thelen, E. (1987). The hole of motor development in developmental psychology: a view of the past and an agenda for the future. In: N. Eisenberg (Ed.). Contemporary topics in developmental psychology. New York: John Wiley \& Sons, 3-33.

23 Thelen, E.; Ulrich, B.D. (1991). Hidden skills: a dynamic systems analysis of treadmill stepping during the first year. Monographs of Society for Research in Child, 56: 1-35.

24 Von Hofsten, C. (1993). Development of goal-directed behavior. In: A. Kalverberg, B. Hopkins, R. Gueze (Eds.). Motor development in early and late childhood. Cambridge: Cambridge University Press, 110-23.

25 Wickstron, R.L. (1977). Fundamental motor patterns. Philadelphia: Lea \& Fabiger.

26 Williams, H. (1983). Perceptual and motor development. New Jersey: Prentice Hall.

27 Williams, K; Haywood K.; Van Sant, A. (1998) Changes in throwing by older adults: a longitudinal investigation. Research Quarterly for Exercise and Sport, 69: 1-10. 